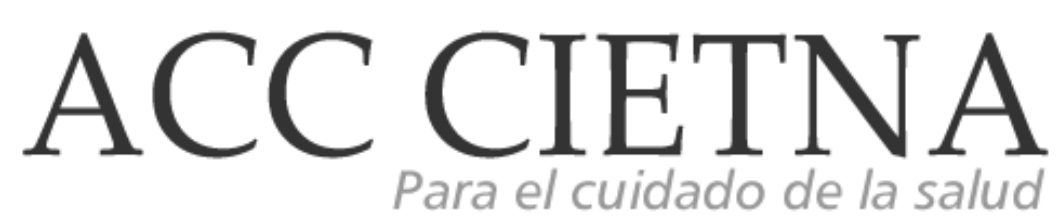

https://doi.org/10.35383/cietna.v4i2.21

\title{
Cotidiano de la persona con ostomía digestiva definitiva -Chiclayo, 2016
}

\author{
Saavedra Olivos Violeta ${ }^{1}$, Sánchez silva Cabrera Andrea de Lourdes ${ }^{2}$, Moncada Espinoza Rosa \\ Leticia $^{3}$
}

\begin{tabular}{l}
\hline INFORMACIÓN DEL ARTÍCULO RESUMEN \\
\hline Historia del artículo:
\end{tabular}

Recibido el 3 de agosto de 2017

Aceptado el 5 de noviembre de 2017

Palabras claves:

Cotidiano

Persona

Ostomía digestiva definitiva

La investigación tuvo como objetivo identificar, describir y analizar el cotidiano de la persona portadora de ostomía digestiva definitiva; se desarrolló en Chiclayo. Las bases conceptuales se sustentaron en los conceptos de cotidiano según Tedesco (1999) y Borgato (2000), persona según Rogers (2012), ostomía digestiva definitiva según Martínez (2014). La metodología fue cualitativa, con abordaje estudio de caso; los sujetos de estudio lo conformaron 10 adultos entre 20 a 80 años que son portadores de ostomía digestiva definitiva por un tiempo igual o mayor de un año y que desearon participar de forma voluntaria en el estudio; utilizándose la entrevista semi-estructurada como técnica de recojo de información. El tamaño de la muestra se determinó con la técnica de saturación y redundancia. El estudio de los datos se efectuó mediante el análisis de contenido según Bardin (2002), respetándose en todo momento los criterios de rigor científico según Castillo (2003) y principios bioéticos de Sgreccia (2007). Los resultados fueron organizados en tres categorías: la vida cotidiana a partir de la ostomía, transformación del cotidiano en el ámbito laboral, social y el apoyo familiar ante el nuevo cotidiano. Se llegó a la conclusión: la persona ostomizada atraviesa por modificaciones en su sentir,

\footnotetext{
${ }^{1}$ Licenciada. Enfermera con trabajo independiente, Chiclayo, Perú.Email: violeta_taleovi@hotmail.com

2 Licenciada. Enfermera con trabajo independiente, Chiclayo, Perú. Email: andreasanchezsilva@hotmail.com .

${ }^{3}$ Magister. Docente de la Escuela de Enfermería de la Universidad Católica Santo Toribio de Mogrovejo, Chiclayo, Perú. Email: rmoncada@usat.edu.pe
} 
necesidades, actividades laborales y relaciones sociales; ocasionadas por las modificaciones en su cuerpo y donde la familia cumple un papel importante en el bienestar y búsqueda de la adaptación ante esta nueva experiencia y reformulación de su cotidiano.

Quotidian person whith definitive digestive ostomy -Chiclayo, 2016

ABSTRACT

Keywords:

Quotidian

Person

Definitive digestive ostomy.
The objective of the research was to identify, describe and analyze the daily life of the person with permanent digestive ostomy; was developed in Chiclayo. The conceptual bases were based on daily concepts according to Tedesco (1999) and Borgato (2000), person according to Rogers (2012), digestive ostomy according to Martinez (2014). The methodology was qualitative, with a case study approach; the study subjects comprised 10 adults between 20 and 80 years who are carriers of definitive digestive ostomy for a time equal to or greater than one year and who wanted to participate voluntarily in the study; using the semi-structured interview as a technique for collecting information. The sample size was determined using the saturation and redundancy technique. The study of the data was carried out through content analysis according to Bardin (2002), respecting at all times the criteria of scientific rigor according to Castillo (2003) and bioethics principles of Sgreccia (2007). The results were organized into three categories: daily life from the ostomy, daily transformation in the social and work environment, and family support for the new daily life. Final conclusion: the ostomized person goes through changes in their feelings, needs, work activities and social relationships; caused by the changes in his body and where the family plays an important role in the well-being and search for adaptation to this new experience and reformulation of their daily life.

\section{Introducción}

La ostomía es un orificio de salida artificial en la pared abdominal producida por diversas causas, que facilita la salida al exterior de los productos de desecho del organismo y puede ser temporal o definitivo'. La colocación de una ostomía de eliminación altera las funciones personales a nivel marital, familiar, laboral, económico y social dando lugar a que surjan múltiples conflictos, pues constituye una ruptura y una crisis en la vida cotidiana de la persona, así mismo supone la adaptación a su nueva imagen. Apareciendo el término "cotidiano de la persona".

Tedesco ${ }^{2}$ defiende la postura que la vida cotidiana es ante todo un producto histórico, donde la define como una dimensión crítica de los procesos sociohistóricos y estructuras que se constituyen en ella. Poniendo a la persona como un ser condicionado por situaciones, necesidades y posibilidades, las 
cuales afectarán y formarán su cotidiano; entendiéndose que el desarrollo del cotidiano depende de los cambios que acontezcan en la sociedad, influyendo esta última en el actuar de la persona y en sus decisiones, es decir, en su modo de vida.

La ostomía digestiva definitiva representa una irrupción violenta y de desconcierto en la vida de la persona, en ella surgen tantos "porqués" y el clásico "cómo podré vivir con esto", reflejándose la intromisión de la enfermedad ante un cotidiano ya establecido; donde la persona se ve obligada a adaptarse a los nuevos cambios que le implica esta nueva experiencia. Cotidiano que al enmarcar la interacción entre la persona y su entorno, muestra un círculo social que se ha visto afectado de alguna manera por la falta de comunicación e información, por el inadecuado manejo de la bolsa de recolección o por la vergüenza a mostrarse con naturalidad ante sus amigos y/o familiares, pudiendo llevar al aislamiento a un grupo de personas y afectar la autoestima de muchas otras.

Frente a la complejidad del tema presentado y a la carencia de estudios a nivel cualitativo, resulta imperativo entender la experiencia de enfrentar la nueva condición de vida de los individuos con ostomía digestiva definitiva; situación que llevo a plantearse el problema de investigación: ¿Cómo es el cotidiano de la persona portadora de ostomía digestiva definitiva en Chiclayo? La investigación tuvo como objeto de estudio: el cotidiano de la persona portadora de ostomía digestiva definitiva.

Diferentes investigaciones de enfermería con relación a la persona ostomizada se han enfocado solamente en el dispositivo, en lo fisiológico; sin llegar a comprender los diferentes modos de reacción de la persona frente a los cambios que experimenta desde el momento de la intervención; por lo que las autoras de esta investigación creyeron necesario profundizar en el "cotidiano" como un espacio colectivo, en el cual la persona actúa, interviene y modifica de acuerdo a situaciones concretas. El cotidiano de la persona portadora de ostomía digestiva definitiva es una temática importante para la profesión de enfermería, en tanto permite captar, conocer y comprender la vida de los sujetos de cuidado, mostrando así la agudeza y conocimiento que se debe impartir en la atención a estos pacientes; de tal forma que el sujeto en su entorno cotidiano configure la información primaria recibida, para construir un esquema de referencia y finalmente concretar un marco de acción transformadora de su realidad, en la cual se reincorporará al ser dado de alta.

El aspecto social se caracteriza porque inspira a mover a la otra persona a que se conduzca de un modo determinado, por lo que crea un contexto de acción supraindividual. Se pueden entender también por actos sociales en sentido amplio, las tomas de posición dirigidas a otras personas, como el amor, el respeto o la admiración, las cuales constituyen una respuesta a valores personales, aunque no necesariamente apuntan a una relación recíproca 3 .

\section{Metodología}

El presente estudio fue una investigación de tipo cualitativa, con abordaje estudio de caso $^{4}$, cuyo objetivo fue desarrollar una descripción minuciosa del objeto de estudio, permitiendo garantizar la fiabilidad en la captación de la realidad de la persona portadora de ostomía digestiva, y su experiencia a partir de los cambios surgidos en un momento dado; dando respuesta al problema de estudio y explicando los resultados del mismo. Así mismo permitió obtener características únicas del individuo con su ambiente, proporcionando una 
mejor comprensión de la temática. Se consideraron como sujetos de investigación a 10 personas que portaban ostomía digestiva definitiva por un tiempo mayor o igual a un año, residentes en Chiclayo. La muestra se seleccionó por conveniencia y fue delimitada, según criterios de saturación y redundancia. Se pudo apreciar que las personas portadoras de ostomía digestiva definitiva cursaban entre los 20 y 80 años, de ambos sexos; quienes aceptaron de forma voluntaria participar en el estudio. El estudio se llevó a cabo en Chiclayo, utilizando la técnica "bola de nieve"; las entrevistas se realizaron en los hogares de las personas con previa cita y coordinación telefónica.

Los datos fueron recolectados mediante la técnica de la entrevista semi-estructurada 5 que constó de dos partes: en la primera se registraron los datos generales, permitiendo caracterizar al personaje entrevistado; y la segunda parte estuvo constituida por seis preguntas abiertas que permitieron conocer el cotidiano de la persona portadora de ostomía definitiva; además se observaron ciertos gestos que formaron parte del sentir de la persona.

El análisis de datos se desarrolló por medio del análisis de contenido ${ }^{6}$, de tal forma que se organizaron los datos a través de 3 fases: el preanálisis, la codificación y la interpretación. En la primera fase, obtenidas las entrevistas se procedió a una primera revisión de las mismas con el fin de identificar los datos más importantes que contribuyan al estudio, así como, testimonios que concurran a un mismo o similar significado; estableciendo un esquema primario.

Durante la segunda fase, se agruparon las unidades de significado por colores, encontrándose códigos, como: los sentimientos que genera la ostomía, la familia, el ámbito social y laboral, también se tomó el aspecto económico y las necesidades que se ven alteradas; y según la relación que existía entre las mismas y al tema al que se referían, se formaron las unidades sintácticas que reagrupándose concluyeron en las tres categorías presentadas. Para finalizar, en la tercera fase, teniendo toda la información ya integrada; y apoyándose en fundamentos teóricos se inició la lectura y relectura para el análisis crítico por parte de las investigadoras, las que se reflejaron en la formulación de las conclusiones.

Durante el desarrollo de la investigación se consideraron y pusieron en práctica los siguientes principios éticos7: defensa de la vida física, principio de libertad y responsabilidad, principio terapéutico o de totalidad, y el de sociabilidad y subsidiaridad. Los entrevistados participaron de forma voluntaria con previa información recibida por parte de las investigadoras, firmando el consentimiento informado. Además de la utilización de seudónimos de flores como: azucena, margarita, gardenia, dalia, acacia, orquídea, tulipán, clavel, girasol y crisantemo; para proteger su identidad; demostrando en todo momento el respeto a su dignidad como ser humano, para finalmente poder publicar los resultados obtenidos bajo anonimato.

Asimismo se tuvieron en cuenta los criterios de rigor científico ${ }^{8}$, con el propósito de asegurar que los hallazgos sean tomados como creíbles y válidos, se establecieron estándares de calidad bajo los siguientes principios: credibilidad, transferibilidad y auditabilidad. El primer principio se evidenció en la base teórica que sustenta el estudio y fue confirmada por las personas que participaron en el mismo, quienes manifestaron su conformidad con los resultados que se obtuvieron.

Para respaldar el segundo principio, las investigadoras describieron con máxima minuciosidad el escenario y los personajes del 
mismo, facilitando así la utilización de los datos de la investigación. Por último, el tercer principio se refiere a la habilidad de otro investigador para seguir la ruta del estudio; por ello se llevó acabo un registro y documentación completa de las decisiones que se tuvieron en cuenta en relación al estudio, resaltando que las encuestas que se recolectaron se conservarán por un tiempo no mayor de 5 años.

\section{Resultados, análisis y discusión}

Después de la recolección y tratamiento de datos, se dio inicio al análisis minucioso de los mismos, obteniendo 3 categorías con sus respectivas subcategorías, de manera que permitieron el ordenamiento y comprensión referida al objeto de estudio. Las cuales se presentan a continuación:

I. La vida cotidiana a partir de la ostomía.

Esta categoría contiene dos subtemas: sentimientos que generó el ser portador de ostomía y cambios en las necesidades de la persona; aquí se evidencia el sentir de la persona frente a las modificaciones en su cuerpo, su calidad de vida y el desarrollo de la misma.

1.1. Sentimientos que generó el ser portador de ostomía.

Los sentimientos son experiencias duraderas, agradables o desagradables, en función de la percepción del estado subjetivo que vive la persona y pueden estar relacionados con actitudes y valores culturales?.

Los sentimientos son bloques de información integrada, síntesis de datos generados por experiencias anteriores, deseos y proyectos, del propio sistema de valores y de la realidad. Son producto de la observación por parte de la mente de los cambios generados por las emociones ${ }^{10}$.

Los sentimientos actúan indirectamente como un medio de adaptación y preparan a la persona para el afrontamiento de un nuevo suceso en su vida; así mismo los sentimientos se evidencian mediante la expresión de tristeza, cólera y frustración, tal y como se muestra en el siguiente discurso:

"Cuando me dijeron que esto debía tenerlo para siempre yo recién tome conciencia y me dije: ¿qué voy a hacer? ¿Cómo voy a hacer para seguir con mi vida?" "Me sentía fastidiada y enojada, me preguntaba ¿por qué a mí? ¿Qué paso? ¿Que hice? ¿Que no hice?" "He tratado de adaptarme a esta nueva forma de vida, al principio me costó trabajo, (...) la ostomía me hace sentir limitada, fastidiada y estresada" "Todo esto me apenaba y fastidiaba, pero ya pues poco a poco lo fui superando; $y$ me decía Dios mío si esto me has mandado pues tengo que aceptarlo" (Azucena)

Este discurso evidencia los múltiples sentimientos por los que ha pasado la persona, estos son experiencias desagradables consecuencia de la situación que enfrenta al ser portador de ostomía; hecho que al ser una interrupción en su vida causó en ella expresiones de fastidio, enojo y limitación; sentimientos que se relacionan con sus deseos y proyectos sin terminar. A su vez al ser expresados y aceptados por la persona, en otras palabras, al ser liberados de alguna forma, encaminan al ostomizado hacia la aceptación de su nuevo cotidiano, apoyándose en su espiritualidad.

La persona portadora de ostomía hace uso de estrategias de afrontamiento para sobrellevar su situación; estas son entendidas como recursos psicológicos que el sujeto pone en marcha para hacer frente a situaciones estresantes. La estrategia de afrontamiento dirigida a la emoción parte de la evaluación en la que el individuo nota 
que no puede hacer nada para modificar las condiciones amenazantes del entorno por lo que recurre a un grupo de procesos cognitivos con los que intenta disminuir la alteración emocional, como son: evitación, minimización, distanciamiento, atención selectiva, comparaciones positivas y la extracción de valores positivos de los sucesos negativos ${ }^{11}$.

En la investigación se encontró sentimientos como la tristeza, la cual es caracterizada por un ánimo bajo y cierto dolor o desplacer; siendo este profundo y prolongado. Su causa es involuntaria y se asocia a la pérdida de alguien a quien amamos o al fracaso ante un valor importante de la vida ${ }^{9}$. La tristeza concibe en el ostomizado una percepción negativa de la vida, llegando a crear desesperanza por no poder cumplir con sus roles con la "normalidad" de la que antes disponían; como se observa en el discurso:

"Me siento triste, ya no soy la misma de antes, es muy difícil adaptarme, lo que me da más tristeza es que ya no puedo cuidar a mis hijas como quisiera: cargarlas, abrazarlas; eso me hace sentir muy mal (Ilora)" (Dalia)

El enojo es la creencia de que la situación no es como debería ser, es decir, la restricción, interferencia o crítica es ilegítima. Proviene de la restricción o limitación, como en la interpretación de que alguna fuerza externa ha interferido con los propios planes, metas o bienestar12. Este sentimiento se produce en la persona ostomizada por el quiebre en su estado de salud, que ha cambiado de manera inesperada sus planes de vida, alterando su bienestar físico y psicológico.

Cabe señalar que en el adulto joven, los sentimientos vertidos representan una señal del proceso insatisfactorio que vivió frente a la modificación física tras la intervención quirúrgica, donde su cotidiano comenzó a reformularse. Esta situación interfiere con el desarrollo en su etapa de vida generándole inseguridad ya que siendo adulto joven debería estar en la cima de sus potencialidades, progreso que se detuvo de forma indefinida a partir de la colocación del estoma.

En la persona ostomizada la cirugía fue un hecho no gestionado, que apareció de manera inesperada y abrupta; generando sentimientos desagradables, siendo estas consecuencias de la transformación del cotidiano y de las condiciones generadas por la presencia del dispositivo. Así como de la percepción que tienen acerca de su propia imagen corporal y las limitaciones que le acarrean, identificándose como personas diferentes.

\subsection{Cambios en las necesidades de la persona.}

La real academia española define el cambio como la acción y efecto de cambiar, la cual se refiere el dejar una cosa o situación para tomar otra ${ }^{13}$. Es la diferencia entre algo o alguien de un momento a otro y es adaptativo en el sentido de que permite lidiar con las condiciones de existencia internas y externas, siempre cambiantes ${ }^{14}$.

La persona portadora de ostomía digestiva definitiva, experimenta diversos cambios que surgen de forma notable a partir de la operación a la que se vio sometida. Dichos cambios se dan tanto a nivel físico como fisiológico, donde la persona puede llegar a perder independencia. Estos han alterado directamente sus necesidades y por ende su calidad de vida. Calvache ${ }^{15}$, señala que la necesidad es entendida como un sistema de condiciones, las cuales, en el organismo humano, contexto cultural y en sus mutuas relaciones en el ambiente, son necesarias y suficientes para la sobrevivencia de un grupo o individuo. 
La necesidad es aquella condición que garantiza la correcta supervivencia, las autoras resumen que dicha condición indica una serie de funciones que la persona debe de cumplir para poder alcanzar un desarrollo correcto y normal; en el caso de la persona ostomizada el proceso abrupto que atraviesa acarrea nuevas necesidades que forman parte de la misma persona, viéndose obligado a adquirir nuevos conocimientos y destrezas para poder enfrentar y lograr adaptarse a un nuevo estilo de vida. Tal y como se narran en los siguientes discursos:

"En comparación con las demás personas que pueden eliminar sus desechos con normalidad, yo tengo que estar mirando que la bolsa no se vaya a derramar" "Muchas veces el olor que emana cuando está lleno el dispositivo es insoportable y si se llega a derramar mancha mi ropa" (Gardenia)

“... entonces yo para salir tengo que comer poco porque no quiero pasar un mal rato, la bolsa se infla y se llena de gases por eso evito comer brócoli, frejoles $y$ alimentos que lo provoquen" "Tengo limitaciones en el comer, me he dado cuenta que comiendo algo restringido cuando tengo que hacer algo en la calle, estoy más tranquila" (Azucena)

La presente investigación hace uso de la pirámide de las 14 necesidades por Maslow ${ }^{16}$ donde se exponen cinco niveles de prioridad, donde el más básico es el primer nivel que incluye necesidades fisiológicas como el aire, agua y la comida; en el segundo contiene a las necesidades de protección y seguridad, lo que implica la seguridad física y psicológica; el tercer nivel comprende necesidades de amor y pertenencia, incluida la amistad, relaciones sociales y el amor sexual; el penúltimo nivel, el cuarto, envuelve necesidades de autoestima y estima, incluyendo la confianza en uno mismo, utilidad, logro y autovaloración; y en el último nivel esta la necesidad de autorrealización, que representa el estado de realización total del potencial, donde la persona tiene la capacidad de resolver los problemas y afrontar las situaciones vitales de forma realista.

La aplicación de la pirámide muestra los diferentes cambios a los que ha sido expuesta la persona portadora de ostomía desde la realización de la cirugía con la posterior integración de la estoma a su vida. La necesidad fisiológica de eliminación es la más afectada pues al estar unida a la intimidad de la persona y debido a la falta de control y llenado sobre el efluente genera en ella sentimientos de inseguridad para reanudar su rutina por la vergüenza y el temor que le ocasiona.

Por otro lado su alimentación se modifica debiendo ajustar su dieta a su nueva condición para que de cierta manera pueda continuar con sus actividades diarias sin molestia alguna. Dentro del cotidiano se ve una transformación en la necesidad de higiene, tomada con mayor minuciosidad y con la incorporación de ciertas habilidades y destrezas para realizarla en su centro de labores o cuando salen a reuniones o viajes. La vestimenta tiende a cambiar pues el ostomizado tiende a usar prendas holgadas; el descanso y sueño también son afectados pues la persona tiene que buscar ciertas posiciones para evitar dañar la bolsa, además de disminuir su descanso al estar al tanto de esta.

Como se evidencia la persona portadora de ostomía digestiva definitiva presenta una serie de alteraciones en sus necesidades, las cuales concluyen en el cambio de su imagen corporal, que lo conduce la adquisición de nuevos conocimientos, habilidades y destrezas para sobrellevarlas pues suponen un gran reto para su vida. 
II. Transformación del cotidiano en el ámbito laboral y social.

En esta segunda categoría se observan las modificaciones socio laborales para la persona ostomizada a partir de la colocación de la ostomía.

\subsection{Repercusión en el ámbito laboral.}

Napolitano ${ }^{17}$ define el ámbito laboral como el espacio o ambiente en donde las personas realizan diferentes labores. Es muy importante destacar la importancia que tiene para una persona estar trabajando en un buen ámbito laboral o ambiente laboral.

De igual manera, Yepes 18 señala que el hombre ama al trabajo cuando siente pasión por crear y poseer lo creado. Por el contrario no lo puede amar cuando es solo fatiga física, esfuerzo, mercancía entregada a cambio de un salario. Cuando el hombre no puede trabajar y llevar a cabo su obra, no alcanza su plenitud.

El cotidiano de la persona ostomizada ha experimentado un rumbo distinto donde la reformulación y/o el abandono de sus trabajos es una realidad encontrada. La persona con ostomía abandona su actividad laboral por el esfuerzo y desgate físico que estas ameritaban, explorando nuevas formas de trabajo desde su hogar o con un empleo independiente que le genera autosuficiencia y actúa como agente distractor. Como se manifestaron en los testimonios:

"Antes tenía dos trabajos pero me vi en la necesidad de dejar uno por mi nueva situación" " $Y$ dado que mis compañeros sabían lo que me había ocurrido me fue más cómodo y fácil reinsertarme en mis labores, sabía que contaba con ellos y esto me daba la tranquilidad de poder usar los servicios higiénicos del establecimiento" (Azucena)
"Yo nunca permitíque esto me limitara, entonces no dejé de trabajar, esto ha sido para mí una experiencia para demostrarle a mi familia que yo sí puedo salir adelante. Ahora como me ve continuo con mi trabajo habitual en el hospital, en lo que es el manejo de las máquinas" (Crisantemo)

Señalando que el ámbito laboral y los personajes que lo componen juegan un rol importante en la adaptación al nuevo cotidiano, rescatando a las relaciones saludables del trabajo como herramientas en las que la persona se apoya, que a su vez destaca la importancia de la comunicación de su nuevo estado de salud para que las personas lo comprendan; igualmente el afrontamiento y superación al ser portador de ostomía dependerá de la madurez emocional, características personales y la actitud tomada por cada individuo.

\subsection{Implicancias sociales en la vida del ostomizado}

Serrano señala que la idea de hombre aislado, ajeno a toda relación con los demás, está en el plano lógico y ontológico en contradicción con la propia naturaleza humana. El hombre necesita a los demás tanto para la realización de sus propios fines biológicos, como para la consecución de sus exigencias económicas, intelectuales, éticas y espirituales ${ }^{19}$.

Es así que todos los actos sociales presuponen un contexto previo de entendimiento, donde las relaciones personales no son actos de una persona, sino algo que existe entre personas $y$ tiene al menos a dos de ellas como portadoras. Se trata de estructuras en cuya constitución desempeñan un cometido en las personas, en sus actos sociales y relaciones sociales ${ }^{20}$.

Por otro lado, en muchas ocasiones la falta de comprensión $\mathrm{y} / \mathrm{o}$ conocimiento por parte de 
quienes conforman el grupo social próximo ocasiona en la persona vergüenza, dicho sentimiento según la literatura tiene que ver con reglas, preceptos, creencias $\mathrm{y} / \mathrm{o}$ valores internalizados sobre uno mismo, sobre sus pensamientos, sentimientos y acciones. Si bien es cierto que uno puede experimentar vergüenza en soledad, no es menos cierto que para su configuración ha sido necesario otro ser cercano y significativo ${ }^{21}$.

Desde que la persona enfrenta la modificación de su cuerpo, toma a la ostomía como un obstáculo para seguir con su vida social. Esta interrupción responde a razones físico - sociales donde influye el comportamiento y actitudes del círculo social ante la persona ostomizada, en quién pueden generar sentimientos de vergüenza e incomprensión. Tal y como muestran los siguientes discursos:

"Ya no salgo a fiestas porque ya no me divierto como antes ya que tengo que estar pendiente de la bolsa, solo asisto a reuniones familiares pues ellos ya conocen mi situación y me comprenden"(Tulipán)

"Hay ocasiones en las que quiero salir a una reunión y no puedo por la vergüenza que me produce tener una bolsa de ostomía, antes participaba en reuniones y me divertía con mis amigos y familiares; pero ahora ya no" (Margarita)

En definitiva físicamente el "portar una bolsa" produce sensación de rechazo, es decir esta respuesta se encuentra relacionada con la aceptabilidad de su nueva condición y del grado de apoyo que se reciba de las personas que lo rodean para que lo ayuden y motiven a seguir adelante. Del mismo modo se señala que la comunicación es importante en la persona ostomizada, ya que al hablar por lo que está pasando y compartir su experiencia, le aporta un sentir de compañía y comprensión. En tal sentido se afirma la idea que los amigos son aquellos que están a tu lado en los momentos más difíciles, para que te ayuden a superarlo y te motiven a seguir adelante.

Rescatando el valor de las relaciones interpersonales, como puente para que la persona ostomizada se reinserte a sus actividades sociales y por ende a la sociedad.

III. El apoyo familiar ante el nuevo cotidiano.

La Organización Mundial de la Salud (OMS) define la familia como los miembros del hogar emparentados entre sí, hasta un grado determinado por sangre, adopción y matrimonio 22 . Por otro lado, Jurado 23 manifiesta que la familia constituye sin lugar a dudas una realidad compleja y multidimensional. Para comenzar, representa una continuidad simbólica que trasciende a cada individuo y generación. En su conformación la familia enlaza tiempo pasado y tiempo futuro, trasmitiendo y preservando celosamente sus señas de identidad. Por esta y otras razones se afirma que la familia es la célula básica de la sociedad.

De igual manera, se menciona que el apoyo proporciona al individuo un sentido de estabilidad y control que lo hace sentirse mejor y percibir de manera más positiva su ambiente; a su vez, esos sentimientos pueden motivarlo a cuidar de sí mismo e interactuar de manera más positiva con las otras personas y a utilizar más recursos personales y sociales para afrontar el estrés ${ }^{24}$.

Una persona integrante de un núcleo familiar comparte y elabora su cotidiano a partir de las relaciones próximas con ellos, y es donde la persona portadora de ostomía se apoya en primera instancia. En ella se resumen también las formas de adaptación y afrontamiento ante situaciones difíciles. Concluyendo que la familia constituye la 
primera escuela para enfrentar la vida y poder superar los retos que esta nos presenten. El apoyo familiar es sinónimo de fuerza y estabilidad, tanto en el carácter económico, emocional e instrumental; siendo estos de vital importancia para su recuperación. De lo mencionado, el apoyo instrumental- económico, se resalta en el paciente ostomizado; ya que como toda enfermedad el poseer una estoma requiere de gastos extras que no estaban presupuestados dentro de la billetera familiar; tal y como se presenta en el siguiente testimonio:

"El hecho de tener la ostomía y la utilización de bolsas e implementos de higiene me generan gastos extras, las bolsas tienen un costo muy alto y los gastos del hogar tuvieron que reajustarse, menos mal que mi hermana y mis sobrinas me brindan su apoyo económico, ellas viven conmigo, y juntas hemos salido adelante" (Azucena)

Siendo la familia el grupo social inmediato, el hombre aprende valores y formas de enfrentar situaciones en su hogar. Para la persona ostomizada la familia va a convertirse en la pieza clave de su adaptación y cuidado, ya que al ser la base educadora proporciona al individuo el impulso para continuar con su desarrollo tanto a nivel personal, físico y social. Con respecto a esto, se manifiesta:

"La relación con mi familia y con mis hijos es buena, ellos me dan la fuerza para seguir viviendo y luchando en esta vida, además mis pequeños nietos me dan esa motivación que necesito para seguir afrontando esta situación" (Margarita)

Se deduce entonces que no solo ocurren cambios en la misma persona, sino que estos cambios afectan a la familia e incluso se afirma que hay un reajuste de ciertos aspectos con la finalidad de ayudar al integrante de la misma a superar esta nueva situación y mostrar la unión familiar que existe y la importancia que esta juega dentro de la sociedad. Concluyendo que la familia constituye el punto de apoyo más importante, necesario y eficaz para la persona portadora de ostomía, encontrando en ella la comprensión, bienestar, auxilio y el impulso para que pueda salir adelante.

\section{Conclusiones}

El cotidiano de la persona ostomizada ha experimentado un rumbo distinto donde la reformulación y/o el abandono de sus trabajos es una realidad presente en la investigación. Por un lado el abandono por el esfuerzo y desgate físico que esta ameritaba, explorando nuevas formas de trabajo que le genera autosuficiencia y actúa como agente distractor. No obstante, es necesario el empoderamiento de la persona ante esta situación, para no limitar sus posibilidades de desarrollo.

Por otro lado, desde que la persona enfrenta la transformación de su cuerpo, toma a la ostomía como un obstáculo para seguir con su vida social. Esta interrupción responde a razones físico sociales donde influye el comportamiento y actitudes que el círculo social adopte, así como la confianza y comunicación que exista entre ambos.

En la investigación se puede destacar que el cotidiano de la persona portadora de ostomía digestiva definitiva ha atravesado un cambio rotundo, donde en primera instancia se encuentran afectados el sentir y la estética física, que lleva al abandono o cambio de actividades laborales y sociales y donde la familia se convierte en un elemento importante en la búsqueda de la adaptación a esta nueva experiencia vivida. 


\section{Bibliografía}

1. Crovari F, Manzor M. Manual De Patología Quirúrgica. Santiago: UC; 2014.

2. Tedesco J. Paradigmas Do Cotidiano: Introdução À Constituição De Um Campo De Análise Social. Santa Cruz: EDURISC; 1999.

3. Aragones J, Amerigo M. Psicología Ambiental. Madrid: Pirámide; 2000.

4. Do Prado M. Investigación Cualitativa En Enfermería: Metodologia y Didáctica. Washington: Organización Panamericana De La Salud; 2013.

5. Burns N, Grove S. Investigación En Enfermería: Desarrollo De La Práctica Enfermera Basada En La Evidencia. $5^{\mathrm{a}}$ Ed. Barcelona: Elsevier; 2012.

6. Bardin L. Análisis de Contenido. $3^{\mathrm{a}}$ ed. Madrid: AKAL; 2002.

7. Sgreccia E. Manual de Bioética: La Bioética y sus Principios. $4^{\text {a }}$ ed. Madrid: Talisio; 2007.

8. Castillo E. El Rigor Metodológico en la Investigación Cualitativa. Colombia: Corporación Editora Médica; 2003.

9. Sanz M, Pollan M, Garrido E. Psicología, Mente y Conducta. $3^{\mathrm{a}}$ ed. Barcelona: Desclee de Brouwer; 2000.

10. Pallares M. Emociones y Sentimientos. España: MARGE BOOKS; 2010.

11. Amarís M, Madariaga $C$, Valle $M$, Zambrano J. Estrategias de afrontamiento individual y familiar frente a situaciones de estrés psicológico. Psicología desde el Caribe. 2013 (consultada 28 abril 2016); 30 (1). Disponible en: http://rcientificas.uninorte.edu.co/index.p $\mathrm{hp} /$ psicologia/article/view/2051/6905
12. Moreno B. Psicología de la Personalidad. Madrid: Thomson; 2007.

13. Real Academia de la Lengua Española. Diccionario de la Lengua Española. 23 ${ }^{\mathrm{a}}$ ed. España: RAE; 2014.

14. Sandoval J. Los procesos de cambio organizacional y la generación de valor. ScienceDirect $\quad 2014 ; \quad 30$. http://www.sciencedirect.com/science/art icle/pii/S0123592314001156 (último acceso 28 abril 2016)

15. Calvache M. La Enfermería SistémicaPropuesta de un Modelo Ecológico. España: Diaz de Santos; 2009.

16. Potter P, Strockert H. Fundamentos De Enfermería. 8 ${ }^{a}$ ed. España: ElServier; 2015.

17. Napolitano G. Motivación En El Ámbito Laboral. España:Babelcube; 2014.

18. Yepes R, Aranguren J. Fundamentos De Antropología. $6^{a}$ ed. España: EUNSA; 2003.

19. Serrano A, Tejedor L. Gestión De Recursos Humanos En Enfermería. España: DiazSantos; 2013.

20. Stein E. La Estructura De La Persona Humana. Madrid: Biblioteca de autores cristianos; 2002.

21. Molina A. Una Mirada Gestáltica Sobre La Vergüenza. Almería: Universidad de Almería; 2014.

22. Clemente C. Definición De Familia Según La OMS.

http://cbtis 149ctsv3lc2.blogspot.pe/2009 /10/definicion-de-familia-segun-laoms.html(último acceso 01 junio 2016)

23. Jurado M. La situación, evolución y composición de las familias vulnerables y su socialización primaria en Matamoros, Tamaulipas. Región Y Sociedad 2016; 28 (65).

http://regionysociedad.colson.edu.mx:80 
85 /index.php/rys/article/view/358

(último acceso 01 junio 2016)

24. Barra E. Apoyo social, estrés y salud.

Psicología y Salud 2004; 14(2). http: / / revistas.uv. $\mathrm{mx} /$ index.php/psicysalu d/article/view/848/1562 (último acceso 01 junio 2016) 